\title{
Time-dependent modelling of particle acceleration and non-thermal emission in Eta Carina
}

\author{
S. Ohm ${ }^{a}$, V. Zabalza ${ }^{* b}$, J. Hinton ${ }^{c}$, E. R. Parkin ${ }^{d}$ \\ ${ }^{a}$ Deutsches Elektronen-Synchrotron (DESY), Zeuthen, Germany \\ ${ }^{b}$ Department of Physics and Astronomy, University of Leicester, University Road, Leicester, LE1 \\ 7RH, United Kingdom \\ ${ }^{c}$ Max-Planck-Institut für Kernphysik, P.O. Box 103980, D 69029 Heidelberg, Germany \\ ${ }^{d}$ School of Physics and Astronomy, University of Leeds, Woodhouse Lane, Leeds LS2 9JT, UK \\ E-mail: stefan.ohmedesy.de, victor.zabalza@le.ac.uk, \\ jim.hinton@mpi-hd.mpg.de
}

Eta Carina is the only colliding-wind binary for which non-thermal emission is detected from hard X-rays to high-energy gamma rays. Although the physical conditions in the shock region change on timescales of hours to days, the variability seen at GeV energies is quite weak and on significantly longer timescales. The gamma-ray spectrum exhibits two spectral features that can be interpreted as emission originating from the shock and post-shock regions of the two distinct stellar winds. Here we report on the first time-dependent modelling of the non-thermal emission in Eta Carina in this scenario, which aims to explain the level of gamma-ray emission as well as the variability with orbital phase. We used a time-dependent acceleration scheme to derive the spectrum of relativistic particles in the adiabatic shock of the companion star, including the effects of cosmic ray pressure modification. We find that emission from primary electrons is unlikely to be responsible for the gamma-ray emission, and that gamma-ray emission from accelerated protons interacting with the dense shocked wind material can explain the observations. The significant pair production absorption in the stellar radiation fields leads to a drop in the predicted gamma-ray emission above several hundred $\mathrm{GeV}$ - consistent with the upper limits as reported by H.E.S.S.

These results have been published in [1].

The 34th International Cosmic Ray Conference,

30 July- 6 August, 2015

The Hague, The Netherlands

${ }^{*}$ Speaker. 


\section{References}

[1] S. Ohm, V. Zabalza, J. A. Hinton, and E. R. Parkin, On the origin of $\gamma$-ray emission in $\eta$ Carina, MNRAS 449 (Apr., 2015) L132-L136, [arXiv: 1502.04056 ]. 